\title{
Effects of Hydroxychloroquine with or without Azithromycin on QT interval in COVID-19: A Systematic Review
}

\author{
Siddharth Shah ${ }^{1}$, Kuldeep Shah ${ }^{2}$, Mohit turagam ${ }^{3}$, Dhanunjaya Lakkireddy ${ }^{4}$, and Jalaj \\ $\operatorname{Garg}^{5}$ \\ ${ }^{1}$ State University of New York Upstate Medical University \\ ${ }^{2}$ West Virginia University \\ ${ }^{3}$ Icahn School of Medicine at Mount Sinai \\ ${ }^{4}$ The Kansas City Heart Rhythm Institute (KCHRI) @ HCA MidWest \\ ${ }^{5}$ Medical College of Wisconsin
}

May 19, 2020

\begin{abstract}
Introduction: Hydroxychloroquine (HCQ) alone or in combination with azithromycin (AZ) is one of the many therapies being explored for the treatment of Coronavirus 2019 (COVID-19). We performed a systematic review regarding the effects of $\mathrm{HCQ}$ versus $\mathrm{HCQ}+\mathrm{AZ}$ on corrected QT interval (QTc) and cardiovascular outcomes. Methods: We performed a systematic search, using PubMed, EMBASE, SCOPUS, and Google Scholar from inception to May 3rd, 2020, with studies fulfilling the following inclusion criteria: (1) compared HCQ versus HCQ+AZ in COVID-19; (2) reported change in QTc interval and/or cardiovascular outcomes. The primary outcome was change in QTc (maximum QTc-baseline QTc) and incidence of TdP in COVID-19 patients on HCQ vs. HCQ+AZ. Results: A statistically significant change in QTc interval was observed with $\mathrm{HCQ}+\mathrm{AZ}$ compared to HCQ alone (WMD $9.13 \mathrm{~ms}$, 95\% CI 3.74-14.01, $\mathrm{p}=0.01, \mathrm{I} 2=29.04 \%$ ). However, no significant difference in the risk of development of QTc $>500$ ms was observed between two groups (10.6\% in HCQ vs. $14.7 \%$ in HCQ+AZ, RR 0.71 , $95 \%$ CI $0.32-1.59, \mathrm{p}=0.40, \mathrm{I} 2=35.8 \%$ ). Also, no significant difference in risk of TdP was observed between the two groups ( $0 \%$ vs. $0.5 \%$, risk difference $-0.002,95 \%$ CI-0.02 to $0.02, \mathrm{p}=0.83, \mathrm{I} 2=0 \%$, respectively). However, one patient experienced TdP, three days after discontinuation of HCQ+AZ for prolonged QTc (499 ms). Conclusion: The risk/benefit of HCQ and AZ should be carefully contemplated, given the risk of QTc prolongation. Until further safety data is available, we recommend close monitoring of QTc interval and electrolytes, avoiding drug-drug interactions in these high-risk patient populations.
\end{abstract}

\begin{abstract}
Introduction : Hydroxychloroquine (HCQ) alone or in combination with azithromycin (AZ) is one of the many therapies being explored for the treatment of Coronavirus 2019 (COVID-19). We performed a systematic review regarding the effects of HCQ versus $\mathrm{HCQ}+\mathrm{AZ}$ on corrected QT interval (QTc) and cardiovascular outcomes.
\end{abstract}

Methods : We performed a systematic search, using PubMed, EMBASE, SCOPUS, and Google Scholar from inception to May $3^{\text {rd }}$, 2020, with studies fulfilling the following inclusion criteria: (1) compared HCQ versus HCQ+AZ in COVID-19; (2) reported change in QTc interval and/or cardiovascular outcomes. The primary outcome was change in QTc (maximum QTc-baseline QTc) and incidence of TdP in COVID-19 patients on HCQ vs. HCQ+AZ.

Results: A statistically significant change in QTc interval was observed with HCQ+AZ compared to HCQ alone (WMD $9.13 \mathrm{~ms}, 95 \%$ CI 3.74-14.01, $\mathrm{p}=0.01, I^{2}=29.04 \%$ ). However, no significant difference in the risk 
of development of QTc $>500 \mathrm{~ms}$ was observed between two groups (10.6\% in HCQ vs. $14.7 \%$ in HCQ+AZ, RR $0.71,95 \%$ CI $0.32-1.59, \mathrm{p}=0.40, I^{2}=35.8 \%$ ). Also, no significant difference in risk of TdP was observed between the two groups ( $0 \%$ vs. $0.5 \%$, risk difference $-0.002,95 \%$ CI- 0.02 to 0.02 , $\mathrm{p}=0.83, I^{2}=0 \%$, respectively). However, one patient experienced TdP, three days after discontinuation of HCQ+AZ for prolonged QTc (499 ms).

Conclusion: The risk/benefit for HCQ and AZ should be carefully contemplated in view of risk of QTc prolongation. Until further safety data is available, we recommend close monitoring of QTc interval and electrolytes, avoiding drug-drug interactions in these high-risk patient populations.

Keywords : Hydroxychloroquine, Azithromycin, Torsades de pointes, COVID-19

Novel Coronavirus 2019 (2019-nCoV, COVID-19), now declared a pandemic, has infected 3.5 million people and killed 243,401 people worldwide ${ }^{1}$. Several pharmacologic approaches have been recommended for the management of COVD-19 infected patients. One such therapy is the use of hydroxychloroquine (HCQ) alone or in combination with azithromycin (AZ). Hydroxychloroquine alone or in combination with azithromycin has a potential for QT prolongation, torsades de pointes (TdP), and sudden cardiac death (SCD). Therefore, to assess the arrhythmic safety, we performed a systematic review regarding the effects of hydroxychloroquine alone versus hydroxychloroquine plus azithromycin on corrected QT interval (QTc) and cardiovascular outcomes in COVID-19 patients.

We performed a systematic search, using PubMed, EMBASE, SCOPUS, and Google Scholar from inception to May $3^{\text {rd }}, 2020$, with studies fulfilling the following inclusion criteria: (1) compared HCQ versus HCQ plus AZ in COVID-19; (2) reported change in QTc interval and/or cardiovascular outcomes; (3) included human subjects; and (4) studies in the English language. The data from included studies were extracted using a standardized protocol and a data extraction form. Any discrepancies were resolved with a consultation with the senior investigator (JG). Two independent reviewers (SS and KS) extracted the following data from the eligible studies: author name, study design, publication year, follow-up duration, number of patients, age, gender, diabetes mellitus (DM), hypertension (HTN), coronary artery disease (CAD), sudden cardiac death, arrhythmias, QT interval, and outcomes. The primary outcome was change in QTc (maximum QTc - baseline QTc) and incidence of TdP in COVID-19 patients on HCQ vs. HCQ + AZ.

Mantel-Haenszel risk ratio (RR) random-effects model (DerSimonian and Laird method) was used to summarize data between the groups ${ }^{2}$. We used the Wan method to estimate the mean and standard deviations ${ }^{3}$. We then calculated the pooled weighted mean difference (WMD) to evaluate the change in QTc interval between the groups. A value of $I^{2}$ of $0-25 \%$ represented insignificant heterogeneity, 26-50\% represented low heterogeneity, $51-75 \%$ represented moderate heterogeneity, and more than $75 \%$ represented high heterogeneity ${ }^{4}$. A two-tailed $\mathrm{p}<0.05$ was considered statistically significant. Statistical analysis was performed using Comprehensive Meta-Analysis version 3.0 (Biostat Solutions, Inc. [BSSI], Frederick, Maryland).

This systematic review of 4 studies [two retrospective ${ }^{5,6}$ and two prospective ${ }^{7,8}$; excluding case reports/series], incorporated a total of 448 patients. The mean age was $60 \pm 13$ years, and $58.7 \%$ were males. Hypertension was the most common comorbidity (56.5\%) followed by diabetes (31.3\%), atrial fibrillation $(10.1 \%)$, coronary artery disease (CAD) (9.6\%), and heart failure (HF) (6.1\%) (Table 1 ). A statistically significant change in QTc interval was observed with HCQ + AZ compared to HCQ alone (weighted mean difference $9.13 \mathrm{~ms}, 95 \%$ CI $3.74-14.01, \mathrm{p}=0.01, I^{2}=29.04 \%$ ) (Figure 1A ). However, no significant difference in the risk of development of QTc $>500 \mathrm{~ms}$ was observed between the two groups (10.6\% in HCQ vs. $14.7 \%$ in HCQ + AZ, RR 0.71, 95\% CI 0.32-1.59, $\mathrm{p}=0.40, I^{2}=35.8 \%$ ) (Figure 1B ). Also, no significant difference in risk of TdP was observed in COVID 19 patients treated alone or with combination drug therapy ( $0 \%$ vs $0.5 \%$, risk difference $-0.002,95 \%$ CI -0.02 to $0.02, \mathrm{p}=0.83, I^{2}=0 \%$, respectively) (Figure 1C).

Drug-induced QT prolongation exhibits 2-3 fold increased risk of TdP (a potentially fatal ventricular arrhythmia); hence are typically avoided if QTc $>500 \mathrm{msec}$. The result of our pooled analysis demonstrated no significant difference in the risk of QTc prolongation $>500 \mathrm{~ms}$ or TdP between the two groups for the treatment of COVID-19 infection. Although QTc $>500 \mathrm{~ms}$ was observed in $13 \%$ patients overall, TdP oc- 
curred in only one patient, three days after discontinuation of HCQ+AZ for prolonged QTc (499 ms). This was likely triggered by additional risk factors, including bradycardia, propofol infusion, cardiomyopathy, and hypothermia ${ }^{6}$. Also, non-sustained ventricular tachycardia (VT) was observed in $2.5 \%$ of patients, while sustained VT occurred in one patient (in the setting of viral myocarditis treated with HCQ) ${ }^{8}$.

Azithromycin has known proarrhythmic effect (regardless of QT prolongation), which is typically dosedependent, likely related to increased intracellular sodium current, with resultant increased risk of SCD. However, only $23 \%$ of patients completed the five-day course for the treatment of COVID-19 infection ${ }^{8}$. Moreover, the prevalence of cardiovascular diseases (such as CAD and HF, which can potentially increase the risk) was low in our pooled analysis. In addition, the risk of drug-induced TdP is higher in critically ill patients (likely due to increased prevalence of other comorbid conditions - advanced age, underlying cardiac disease, electrolyte imbalance, drug-drug interaction, and genetics (as evident in one patient who had TdP despite drug discontinuation). Furthermore, patients have an increased risk of ventricular arrhythmias from COVID-19 disease itself (likely secondary to cardiomyopathy and myocarditis). These factors alone or in combination, suggest the possibility of underestimating the risk of TdP and SCD in our pooled analysis (also evident by the fact that only $15.6 \%$ of patients in our study were treated in an intensive care unit setting).

The risk of TdP may be higher in such vulnerable populations (critically ill patients at risk for cardiomyopathy and myocarditis, electrolyte derangements, drug-drug interactions, and those with history of cardiovascular diseases), where extreme caution is warranted. Therefore, risk/benefit for HCQ and AZ should be carefully contemplated. Until further safety data is available, we recommend close monitoring of QTc interval and electrolytes, avoiding drug-drug interactions in these high-risk patient populations. There is a critical need for larger randomized controlled studies to assess the risk/benefit profile of these drugs in COVID-19 therapy.

\section{References}

1. Coronavirus disease (COVID-19) Situation Report- 106. https://www.who.int/docs/defaultsource/coronaviruse/situation-reports/20200505covid-19-sitrep-106.pdf?sfvrsn=47090f63_2. Accessed 14th May 2020

2. DerSimonian R, Laird N. Meta-analysis in clinical trials. Control Clin Trials. 1986;7(3):177-188.

3. Wan X, Wang W, Liu J, Tong T. Estimating the sample mean and standard deviation from the sample size, median, range and/or interquartile range. BMC Med Res Methodol. 2014;14:135.

4. Higgins JP, Altman DG, Gotzsche PC, et al. The Cochrane Collaboration's tool for assessing risk of bias in randomised trials. BMJ. 2011;343:d5928.

5. Bessiere F, Roccia H, Deliniere A, et al. Assessment of QT Intervals in a Case Series of Patients With Coronavirus Disease 2019 (COVID-19) Infection Treated With Hydroxychloroquine Alone or in Combination With Azithromycin in an Intensive Care Unit.JAMA Cardiol 2020 [E-pub ahead of print] https://dx.doi.org/10.1001/ jamacardio.2020.1787.

6. Mercuro NJ, Yen CF, Shim DJ, et al. Risk of QT Interval Prolongation Associated With Use of Hydroxychloroquine With or Without Concomitant Azithromycin Among Hospitalized Patients Testing Positive for Coronavirus Disease 2019 (COVID-19). JAMA Cardiol. 2020 [E-pub ahead of print], https://dx.doi.org/10.1001/jamacardio.2020.1834.

7. Saleh M, Gabriels J, Chang D, et al. The Effect of Chloroquine, Hydroxychloroquine and Azithromycin on the Corrected QT Interval in Patients with SARS-CoV-2 Infection. Circ Arrhythm Electrophysiol.2020. [E-pub ahead of print], https://dx.doi.org/10.1161/ CIRCEP.120.008662.

8. Chang D, Saleh M, Gabriels J, et al. Inpatient Use of Ambulatory Telemetry Monitors for COVID-19 Patients Treated with Hydroxychloroquine and/or Azithromycin. J Am Coll Cardiol. 2020 . [E-pub ahead of print], https://dx.doi.org/10.1016/j.jacc.2020.04.032. 


\section{Figure Legends}

Table 1: Baseline characteristics of studies included in our meta-analysis

Figure 1: A - The forest plot for pooled weighted mean difference (WMD) for change in QTc interval (maximum QTc - baseline QTc) in COVID-19 patients treated with hydroxychloroquine (HCQ) vs. hydroxychloroquine + Azithromycin (HCQ + AZ); B - The forest plot for risk of QTc prolongation > $500 \mathrm{~ms}$ in COVID-19 patients treated with hydroxychloroquine (HCQ) vs. hydroxychloroquine + Azithromycin (HCQ $+\mathrm{AZ}) ; \mathbf{C}$ - The forest plot for risk of TdP in COVID-19 patients treated with hydroxychloroquine (HCQ) vs. hydroxychloroquine + Azithromycin ( $\mathrm{HCQ}+\mathrm{AZ})$.

Table 1 Baseline characteristics of studies included in our pooled analysis.

\begin{tabular}{lllllllll}
\hline Study name & Study type & Year & Country & N & Age (years) (mean \pm SD) & Male & Hypertension & Diabet \\
\hline Saleh et al & Prospective & 2020 & USA & 201 & $58.5 \pm 9.1$ & 115 & 121 & 65 \\
Chang et al & Prospective & 2020 & USA & 117 & $60.2 \pm 14.9$ & 70 & 61 & 33 \\
Bessiere et al & Retrospective & 2020 & France & 40 & $68($ IQR 58-74) (median) & 32 & 23 & 16 \\
Mercuro et al & Retrospective & 2020 & USA & 90 & $60.1 \pm 16.1$ & 46 & 48 & 26 \\
\hline
\end{tabular}

$\mathrm{SD}$ - standard deviation; IQR - interquartile range; NR - not reported.

Figure 1

(A) Change in QTc interval

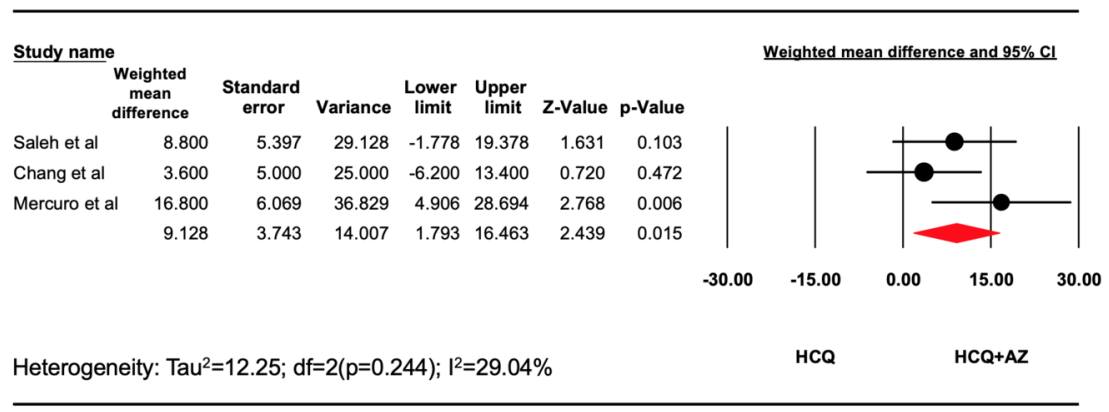

(B) Risk of QTC prolongation $>500 \mathrm{~ms}$

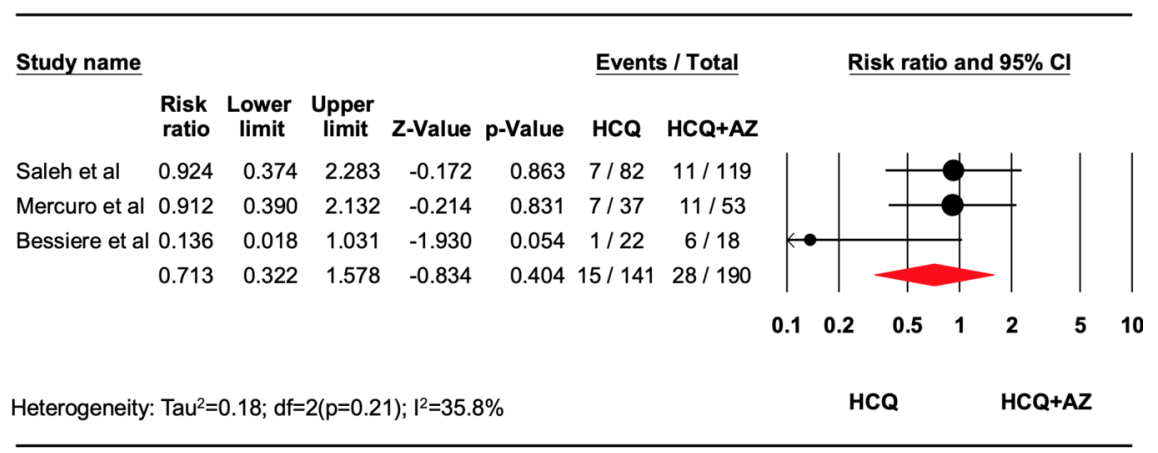


(C) Risk of Torsades de pointes

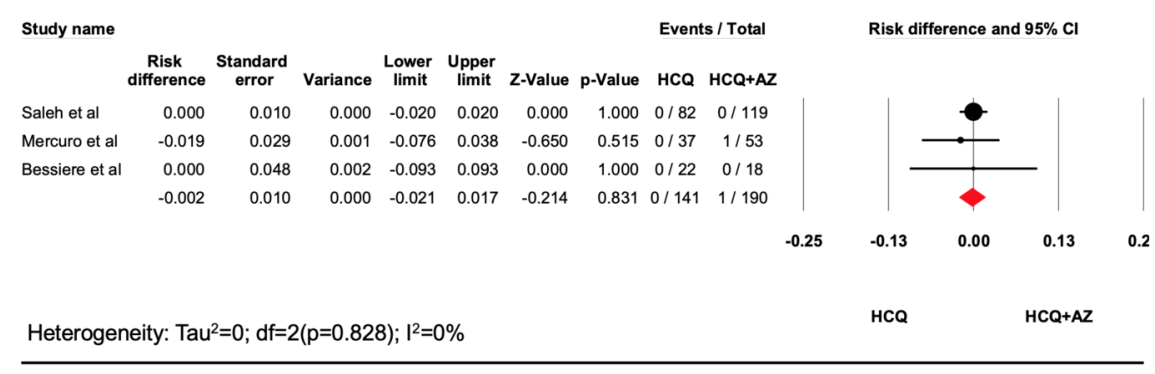

